\title{
Topological generation results for free unitary and orthogonal groups
}

\author{
Alexandru Chirvasitu
}

\begin{abstract}
We show that for every $N \geq 3$ the free unitary group $U_{N}^{+}$is topologically generated by its classical counterpart $U_{N}$ and the lower-rank $U_{N-1}^{+}$. This allows for a uniform inductive proof that a number of finiteness properties, known to hold for all $N \neq 3$, also hold at $N=3$. Specifically, all discrete quantum duals $\widehat{U_{N}^{+}}$and $\widehat{O_{N}^{+}}$are residually finite, and hence also have the Kirchberg factorization property and are hyperlinear. As another consequence, $U_{N}^{+}$are topologically generated by $U_{N}$ and their maximal tori $\widehat{\mathbb{Z}^{* N}}$ (dual to the free groups on $N$ generators) and similarly, $O_{N}^{+}$are topologically generated by $O_{N}$ and their tori $\widehat{\mathbb{Z}_{2}^{* N}}$.

Key words: compact quantum group, free unitary group, free orthogonal group, torus, topological generation, residually finite, hyperlinear, Kirchberg factorization property
\end{abstract}

MSC 2010: 20G42; 16T20; 46L52

\section{Introduction}

The compact quantum group literature has recently seen considerable interest in the notion of topological generation (e.g. [9, 4, 2, 3]). The term was coined in [9], and the concept naturally extends its classical counterpart, applicable to ordinary compact groups:

Let $H, K \leq G$ be two closed subgroups of a compact group. Let also $\mathcal{O}(-)$ denote the algebra of representative functions on a compact group (i.e. the algebra generated by matrix entries of finitedimensional representations on Hilbert spaces). Then, $G$ is the closure of the subgroup generated by $H$ and $K$, written

$$
G=\langle H, K\rangle,
$$

if and only if there is no proper quotient $*$-Hopf algebra $\mathcal{O}(G) \rightarrow \mathcal{A}$ through which both

$$
\mathcal{O}(G) \rightarrow \mathcal{O}(H) \quad \text { and } \quad \mathcal{O}(G) \rightarrow \mathcal{O}(K)
$$

factor.

Formally, the compact quantum groups in this paper are objects dual to the CQG algebras of [12]: cosemisimple complex Hopf $*$-algebras $\mathcal{A}$ with positive unital integral $h: \mathcal{A} \rightarrow \mathbb{C}$. These are to be thought of as algebras $\mathcal{O}(G)$ of representative functions on the corresponding compact quantum group. Given that classically topological generation can be cast in function-algebra terms, the notion carries over to the quantum setting (this is a paraphrase of [9, Definition 4]):

Definition 0.1 Let $G_{i} \leq G, i \in I$ be a family of quantum subgroups of a compact quantum group. The family topologically generates $G$, written as

$$
G=\left\langle G_{i}, i \in I\right\rangle
$$


if the quotients

$$
\mathcal{O}(G) \rightarrow \mathcal{O}\left(G_{i}\right)
$$

do not factor through any proper quotient Hopf $*$-algebra of $\mathcal{O}(G)$.

Here (and throughout the paper) 'quantum subgroups' means quotient Hopf $*$-algebra.

The notion of topological generation appears under different terminology in [10]. Specifically, [10, Defintiion 2.15] introduces the concept of a jointly full family of functors $\mathcal{C} \rightarrow \mathcal{C}_{i}$. In that language, $G_{i}$ topologically generate $G$ precisely when the scalar corestriction functors

$$
\mathcal{M}^{\mathcal{O}(G)} \rightarrow \mathcal{M}^{\mathcal{O}\left(G_{i}\right)}
$$

between the respective categories of comodules form a jointly full family.

As yet another characterization, the condition is equivalent to the requirement that the morphism

$$
\mathcal{O}(G) \rightarrow \prod^{C Q G} \mathcal{O}\left(G_{i}\right)
$$

to the product in the category of CQG algebras (not the product of underlying algebras!) resulting from the maps (1) is an embedding.

In the language of [7, Definition 1.12], [10, Theorem 3.1] shows that the Pontryagin duals to the free unitary and orthogonal groups $U_{N}^{+}$and $O_{N}^{+}, N \geq 2$ are residually finite provided $N \neq 3$ (see Section 1 below for a reminder on these objects). The reason for the gap is that the argument proceeds inductively, using the following topological generation result ([10, Lemma 3.13 and Remark $3.14])$ :

Theorem 0.2 For all $N \geq 4$ we have

$$
U_{N}^{+}=\left\langle U_{N}, U_{N-1}^{+}\right\rangle
$$

Similarly, the case $N=3$ is problematic in $[9,8]$ for reasons ultimately traceable to the same phenomena (see e.g. [9, Remark 5]).

One of the main goals of the present note is to extend Theorem 0.2 to $N=3$ in Proposition 2.1 below. This will then have a number of consequences:

- First off, the results of $[9,10,8]$ extend to $N=3$.

- Secondly, we obtain in Section 3 results to the effect that for all $N \geq 2$ the free unitary and orthogonal quantum groups $U_{N}^{+}$and $O_{N}^{+}$are topologically generated by their "maximal tori".

The underlying Hopf algebra $\mathcal{O}\left(U_{N}^{+}\right)$is generated as a $*$-algebra by generators $u_{i j}, 1 \leq i, j \leq N$. Annihilating the off-diagonal generators

$$
u_{i j}, 1 \leq i \neq j \leq N
$$

produces a quotient Hopf algebra isomorphic to the group algebra $\mathcal{O}\left(\mathbb{T}_{N}^{+}\right)$of the free group on $N$ generators (the images of the diagonal $u_{i i}$ ). This is the non-commutative analogue of the function algebra on the "standard torus" embedded diagonally in the unitary group $U_{N}$, and the second bullet point above is a paraphrase of Theorem 3.1, that $U_{N}^{+}$is generated by all of the conjugates of $\mathbb{T}_{N}^{+}$by the elements of the classical subgroup $U_{N}<U_{N}^{+}$.

The orthogonal picture provided by Theorem 3.3 is similar, the analogue of the maximal torus $\mathbb{T}_{N}^{+}$this time being the Pontryagin dual of the free product $\mathbb{Z}_{2}^{* N}$ of $N$ copies of $\mathbb{Z}_{2}$. 
The study of maximal tori in compact quantum groups $G$ (meaning maximal cocommutative Hopf $*$-algebra quotients of $\mathcal{O}(G)$ ) was initiated in [6] and [3] treats torus-generation themes similar to some the present paper's contents.

Section 1 briefly recalls some of the relevant background.

In Section 2 we prove Proposition 2.1, extending the topological generation result in Theorem 0.2 to the case $N=3$.

Finally, Section 3 records the torus-generation consequences alluded to above, in Theorems 3.1 and 3.3 .

\section{Acknowledgements}

This work was partially supported by NSF grant DMS-1801011.

I am grateful for numerous stimulating conversations with Teodor Banica (who has been very generous in sharing his problems), Michael Brannan and Amaury Freslon.

\section{Preliminaries}

We assume some Hopf algebra background; [17, 1, 15, 16] are all good sources. For the purposes of this paper compact quantum groups appear in their CQG algebra guise, as in [12, Definition 2.2]. An equivalent characterization of those objects reads as follows.

Definition 1.1 A $C Q G$ algebra is a complex Hopf $*$-algebra $\mathcal{A}$ with the following properties:

- $\mathcal{A}$ is cosemisimple, i.e. its category of (either left or right) comodules is semisimple.

- the unique unital left and right integral ([15, Definition 2.4.4 and Theorem 2.4.6]) $h: \mathcal{A} \rightarrow \mathbb{C}$ is positive, in the sense that $h\left(x^{*} x\right) \geq 0$ for all $x \in \mathcal{A}$.

The category of compact quantum groups is the category dual to that of CQG algebras. We write $\mathcal{O}(G)$ for the Hopf algebra attached to a compact quantum group $G$.

We occasionally refer to compact quantum groups as 'quantum groups', the phrase being unambiguous throughout this note.

Example 1.2 The preeminent examples in our context are algebras $\mathcal{O}(G)$ of representative functions on compact groups $G$. $h$ is then simply integration against the Haar measure on $G$, justifying the terminology of Haar state for the functional $h$ from Definition 1.1.

Example 1.3 For $N \geq 2$ the free unitary group $U_{N}^{+}$is the compact quantum group whose underlying CQG algebra $\mathcal{O}\left(U_{N}^{+}\right)$is generated by $u_{i j}, 1 \leq i, j \leq N$ as a $*$-algebra, with relations demanding that both

$$
u:=\left(u_{i j}\right)_{i, j} \quad \text { and } \quad \bar{u}:=\left(u_{i j}^{*}\right)_{i, j} \in M_{N}(\mathcal{A})
$$

are unitary.

The Hopf algebra structure is given by comultiplication and counit

$$
\Delta: u_{i j} \mapsto \sum_{k} u_{i k} \otimes u_{k j} \quad \text { and } \quad \varepsilon: u_{i j} \mapsto \delta_{i j}
$$

respectively, where $\delta_{i j}$ denotes the Kronecker delta. 
Example 1.4 Let $N \geq 2$ again. The free orthogonal group $O_{N}^{+}$has associated Hopf algebra $\mathcal{O}\left(O_{N}^{+}\right)$ as in Example 1.3, with the additional constraints that the generators $u_{i j}$ be self-adjoint.

Examples 1.3 and 1.4 are central to the discussion below. The objects were introduced in [18], there the respective Hopf algebras were denoted by $A_{u}(N)$ and $A_{o}(N)$. Similarly, we have the following construction introduced in [19].

Example 1.5 For $N \geq 2$ the free symmetric group $S_{N}^{+}$has CQG algebra $\mathcal{O}\left(S_{N}^{+}\right)$generated by the self-adjoint idempotent elements $u_{i j}$ (i.e. projections) such that the sums across the rows and columns of the matrix $u=\left(u_{i j}\right)_{i, j}$ in $M_{N}\left(\mathcal{O}\left(S_{N}^{+}\right)\right)$are all equal to $1 \in \mathcal{O}\left(S_{N}^{+}\right)$.

Example 1.6 For every discrete group $\Gamma$ the group algebra $\mathbb{C} \Gamma$ is a $C Q G$ algebra when equipped with the $*$-structure making all $\gamma \in \Gamma$ unitary and with the comultiplication and counit

$$
\Delta: \gamma \mapsto \gamma \otimes \gamma \quad \text { and } \quad \varepsilon: \gamma \mapsto 1
$$

Conceptually, the compact quantum group attached to this CQG algebra should be thought of as the Pontryagin dual of $\Gamma$, for which reason we write $\mathbb{C} \Gamma=\mathcal{O}(\widehat{\Gamma})$.

Motivated by Example 1.6 we write, as is customary in the field, $\widehat{G}$ for the discrete quantum group" dual to $G$. For our purposes, no separate definition of a discrete quantum group is necessary: $\widehat{G}$ is to be thought of simply as a virtual object whose underlying "group algebra" $\mathcal{O}(G)$.

We will also refer frequently to representations of a compact quantum group:

Definition 1.7 Let $G$ be a compact quantum group. Its category of representations is the category $\mathcal{M}^{\mathcal{O}(G)}$ of $\mathcal{O}(G)$-comodules.

Just as is the case for an ordinary compact group, representations form a monoidal category. Furthermore, the finite-dimensional representations form a rigid monoidal category: every object $V$ has a dual $V^{*}$ and the canonical evaluation map $V^{*} \otimes V \rightarrow \mathbb{C}$ is a morphism in $\mathcal{M}^{\mathcal{O}(G)}$ to the trivial object $\mathbb{C}$. We refer to [13, Chapter 2] for background on the topic.

\section{Free unitary groups in small degree}

As recalled in the introduction, one of the results of [10] is the fact that for all $N \geq 4$ we have

$$
U_{N}^{+}=\left\langle U_{N}, U_{N-1}^{+}\right\rangle:
$$

see [10, Lemma 3.13 and Remark 3.14].

The first aim of the present note is to observe that in fact the proof of [10, Lemma 3.13] can be slightly altered so as to also allow (2) for $N=3$ :

Proposition 2.1 For all $N \geq 3$ we have

$$
U_{N}^{+}=\left\langle U_{N}, U_{N-1}^{+}\right\rangle
$$

Let $V$ be the fundamental representation of $U_{N}^{+}$. Reprising the notation of [10], for a tuple $\left(\varepsilon_{i}\right)_{i=1}^{k}$ consisting of blanks and ' $*$ ' we set

$$
V^{\left(\varepsilon_{i}\right)}:=V^{\varepsilon_{1}} \otimes \cdots \otimes V^{\varepsilon_{k}}
$$


(i.e. a tensor product of copies of $V$ and its dual $V^{*}$ ).

The representation theory of $U_{N}^{+}$is well understood, having been worked out in [5]. In particular, every $U_{N}^{+}$-intertwiner

$$
V^{\left(\varepsilon_{i}\right)} \rightarrow \mathbb{C}
$$

is a linear combination of non-crossing partitions: evaluation of each copy of $V^{*}$ against one copy of $V$ in such a manner that strings connecting the $\left(V^{*}, V\right)$ pairs can be drawn in the plane so as not to intersect (and all $V$ and $V^{*}$ get paired off).

Proof of Proposition 2.1 As noted above, [10, Lemma 3.13] already takes care of the case $N \geq 4$ so we assume $N=3$. We will examine the proof of that result and amplify it appropriately.

In the proof in question, one considers a tensor product $V^{\left(\varepsilon_{i}\right)}$ of the fundamental representation of $U_{N}^{+}$and seeks to argue that a linear map $f: V^{\left(\varepsilon_{i}\right)} \rightarrow \mathbb{C}$ is a $U_{N}^{+}$-intertwiner provided it is an intertwiner over both $U_{N}$ and $U_{N-1}^{+}$.

In turn, the hypothesis on $f$ is reinterpreted as follows: for every decomposition $V=W \oplus U$ with

$$
\operatorname{dim} W=N-1, \quad \operatorname{dim} U=1
$$

the restriction of $f$ to every summand of the form

$$
Z^{\left(\varepsilon_{i}\right)} \subset V^{\left(\varepsilon_{i}\right)}, \quad Z \in\{W, U\}
$$

is a linear combination of non-crossing pairings between $W, W^{*}$ and $U, U^{*}$ tensorands. The conclusion would then have to be that $f$ is a span of non-crossing pairings $V^{*} \otimes V \rightarrow \mathbb{C}$.

By moving the complement $U$ of $W$ continuously, we can furthermore assume that $W$ and $U$ are in arbitrary position: $U$ can be contained in $W$ as well as complementary to it. Furthermore, by subtracting an appropriate span of non-crossing pairings from $f$ we can assume that

$$
\left.f\right|_{W^{\left(\varepsilon_{i}\right)}} \cong 0
$$

the desired conclusion would then be that $f$ vanishes identically on $V^{\left(\varepsilon_{i}\right)}$.

We now proceed essentially as in the proof of [10, Lemma 3.13]. Consider the restriction of $f$ to a subspace of the form (3) for a one-dimensional complement $U$ of $W$. Let $\left(\omega_{j}\right)$ be the sub-tuple of $\left(\varepsilon_{i}\right)$ consisting of those $i$ for which the $i^{\text {th }}$ tensorand $Z$ in $V^{\left(\varepsilon_{i}\right)}$ is $W$ (i.e. the $(N-1)$-dimensional subspace rather than the 1-dimensional one). If $e$ and $e^{*}$ are mutually dual bases of $U$ and $U^{*}$ respectively, let

$$
\psi: W^{\left(\delta_{j}\right)} \rightarrow Z^{\left(\varepsilon_{i}\right)}
$$

be the map obtained by inserting $e$ and $e^{*}$ in the spots deleted in passing from (varepsilon $i$ ) to $\left(\delta_{j}\right)_{j}$ (see Example 2.2). Then, the restriction of $f$ to $Z^{\left(\varepsilon_{i}\right)}$ is uniquely determined by $f \circ \psi$, and the latter is a linear combination

$$
\sum_{\pi} c_{\pi} \pi
$$

of non-crossing pairings $\pi$ of $W$ and $W^{*}$ tensorands in $W^{\left(\delta_{j}\right)}$. Because $f$ is a $U_{N}$-intertwiner, the same linear combination (4) is valid for any choice of $W$ and $U$, including, as observed above, the non-complementary case of $U \subseteq W$. But in that case $f=0$ because $f$ vanishes on $W^{\left(\varepsilon_{i}\right)}$, meaning that (4) is identically zero.

The linear independence of the non-crossing pairings on an $(N-1)$-dimensional space $W$ for $N-1=2$ now finishes the proof. 
Example 2.2 If, say,

$$
Z^{\left(\varepsilon_{i}\right)}=W \otimes U \otimes U^{*} \otimes W^{*}
$$

then

$$
W^{\left(\delta_{j}\right)}=W \otimes W^{*} \text { and } \psi: W \otimes W^{*} \rightarrow W \otimes U \otimes U^{*} \otimes W^{*}
$$

is the map

$$
W \otimes W^{*} \ni w \otimes w^{*} \mapsto w \otimes e \otimes e^{*} \otimes w^{*} \in W \otimes U \otimes U^{*} \otimes W^{*} .
$$

\subsection{Residual finiteness, hyperlinearity and factorization}

As noted briefly in the introductory discussion above, Proposition 2.1 allows us to extend some of the finiteness results in the literature to $U_{3}^{+}$and $O_{3}^{+}$. This requires that we recall some terminology. The following notion appears in [7, Definition 1.12].

Definition 2.3 Let $G$ be a compact quantum group. The corresponding discrete quantum group $\widehat{G}$ is residually finite if $\mathcal{O}(G)$ is finitely generated and embeds as a $*$-algebra in a product of matrix algebras.

With this in hand, we have

Theorem 2.4 One can simply reprise the proof of [10, Theorem 3.1], in light of the new information provided by Proposition 2.1 at $N=3$.

For all $N \geq 2$ the discrete quantum groups $\widehat{U_{N}^{+}}$and $\widehat{O_{N}^{+}}$are residually finite.

Proof [10, Corollary 2.16] says that residual finiteness for discrete quantum groups $\widehat{G}$ is inherited from families $\widehat{G_{i}}$ if

$$
G=\left\langle G_{i}\right\rangle
$$

is a topologically generating family. [10, Lemma 3.9] proves the claim for $\widehat{U_{2}^{+}}$, and since residual finiteness also holds for duals $\widehat{U_{N}}$ of classical unitary groups the conclusion in the unitary case follows inductively from Proposition 2.1.

As for the orthogonal claim, it is equivalent to the unitary counterpart by [10, Proposition $3.8]$.

Residual finiteness, in turn, entails other properties of interest in the literature. The Connes embedding problem (CEP for short) raised in [11] has driven much of the development in operator algebras. It asks whether every finite von Neumann algebra $N$ with separable predual $N_{*}$ equipped with a trace $\tau$ embeds in a trace-preserving fashion in an ultrapower $R^{\omega}$ of the hyperfinite $I I_{1}$ factor $R$ with respect to some ultrafilter $\omega$ on $\mathbb{N}$.

A discrete group is hyperlinear when its GNS von Neumann algebra with respect to the standard trace satisfies CEP. Motivated by this, we have [9, §3.2].

Definition 2.5 A discrete quantum group $\widehat{G}$ is hyperlinear if the GNS von Neumann algebra associated to $\mathcal{O}(G)$ equipped with the state $h$ satisfies CEP.

Remark 2.6 Note that the discussion in Definition 2.5 is only meaningful for Kac type quantum groups, i.e. those for which the Haar state $h$ is tracial: $h(x y)=h(y x)$ for all $x, y \in \mathcal{O}(G)$.

The following result extends [9, Corollary 4.3, Theorem 4.4] to $N=3$. 
Corollary 2.7 The discrete duals $\widehat{U_{N}^{+}}$and $\widehat{O_{N}^{+}}$are hyperlinear for all $N \geq 2$.

Proof This follows from Theorem 2.4: as in the case of ordinary discrete groups, residual finiteness entails hyperlinearity (see Remark 2.10 below).

We next we turn to the Kirchberg factorization property, introduced in [14] for discrete groups and studied more generally in the context of discrete quantum groups in [8, 7] (see [8, Theorem 28 and Definitions 2.9 and 2.10]).

Definition 2.8 A discrete quantum group $\widehat{G}$ has the Kirchberg factorization property if the natural action of the algebraic tensor product $\mathcal{O}(G) \otimes \mathcal{O}(G)^{o p}$ on the GNS Hilbert space $L^{2}(G, h)$ with respect to the Haar stat extends to the minimal $C^{*}$ tensor product of the two enveloping $C^{*}$ algebras.

Once more, the bulk of the following result known: all cases $N \neq 3$ are covered by [8, Theorems 4.3 and 4.4$]$.

Corollary 2.9 All $\widehat{U_{N}^{+}}$and $\widehat{O_{N}^{+}}, N \geq 2$ have the Kirchberg factorization property.

Proof This is again a consequence of Proposition 2.1: by [7, Theorem 2] residual finiteness implies the Kirchberg factorization property.

Remark 2.10 The proof of Corollary 2.9 also sheds some light on that of Corollary 2.7: as observed in [8, Remark 2.11], the factorization property implies hyperlinearity. Together with [7, Theorem 2] this justifies the claim made in the proof of Corollary 2.7 that residual finiteness does too, and shows that the three properties discussed above are ordered by strength as follows:

$$
\text { residual finiteness } \Rightarrow \text { factorization property } \Rightarrow \text { hyperlinearity. }
$$

\section{An application to generation by tori}

Assuming Proposition 2.1, we propose to address the following problem. Denote

$$
T_{N}^{+}=\widehat{\mathbb{Z}_{2}^{* N}}, \quad \mathbb{T}_{N}^{+}=\widehat{\mathbb{Z}^{* N}}
$$

Then, we have

Theorem 3.1 For all $N \geq 2$,

$$
U_{N}^{+}=\left\langle U_{N}, \mathbb{T}_{N}^{+}\right\rangle
$$

Proof We do this by induction on $N$.

Induction step: $N \geq 3$. We know from Proposition 2.1 that

$$
U_{N}^{+}=\left\langle U_{N}, U_{N-1}^{+}\right\rangle
$$

and by the induction hypothesis

$$
U_{N-1}^{+}=\left\langle U_{N-1}, \mathbb{T}_{N-1}^{+}\right\rangle .
$$

The conclusion now follows from $\mathbb{T}_{N-1}^{+}<\mathbb{T}_{N}^{+}$. 
Base case: $N=2$. According to [5, Lemme 7] we have a surjection

$$
\mathbb{T} \hat{*} S U_{2} \rightarrow U_{2}^{+}
$$

(in the sense that the opposite morphism of Hopf algebras is an inclusion). Since the left hand side is generated by $\mathbb{T} \hat{*} \mathbb{T}$ and $S U_{2}$ (by Theorem 3.4 applied to $G=S U_{2}$ ), the right hand side will be generated by the images $\mathbb{T}_{2}^{+}$and $S U_{2} \subset U_{2}$ of these two quantum groups through (5).

Corollary 3.2 For all $N \geq 2$,

$$
U_{N}^{+}=\left\langle O_{N}, \mathbb{T}_{N}^{+}\right\rangle
$$

Proof This follows from Theorem 3.1 and the fact that $U_{N}$ is topologically generated by $O_{N}$ and $\mathbb{T}_{N}<\mathbb{T}_{N}^{+}$.

As a consequence, we have the analogous orthogonal result:

Theorem 3.3 For all $N \geq 2$,

$$
O_{N}^{+}=\left\langle O_{N}, T_{N}^{+}\right\rangle
$$

Proof First, consider the projectivization $P O_{N}^{+}$whose underlying *-algebra is generated by

$$
u_{i j}^{*} u_{k l}=u_{i j} u_{k l}
$$

and the unitary analogue $P U_{N}^{+}$. The inclusion $O_{N}^{+} \rightarrow U_{N}^{+}$induces an isomorphism

$$
P O_{N}^{+} \cong P U_{N}^{+}
$$

(e.g. by [5, Théorème 1] or [10, Proposition 3.3]). Since Corollary 3.2 ensures that

$$
P O_{N}^{+}=P U_{N}^{+}=\left\langle P O_{N}, P \mathbb{T}_{N}^{+}\right\rangle=\left\langle P O_{N}, P T_{N}^{+}\right\rangle
$$

we at least know that (6) holds "projectively".

Now let

$$
G=\left\langle O_{N}, T_{N}^{+}\right\rangle \leq O_{N}^{+}
$$

Since the center $\mathbb{Z}_{2}<O_{N}^{+}$is contained in $O_{N}$ and $T_{N}^{+}$, we have a commutative diagram

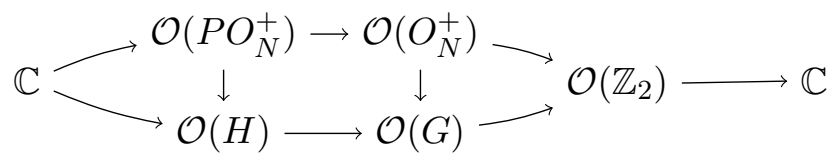

of Hopf algebras with exact rows, surjective columns, and

$$
H=\left\langle P O_{N}, P T_{N}^{+}\right\rangle \leq P O_{N}^{+}
$$

Because $H=P O_{N}^{+}$by (7) the left hand vertical arrow is an isomorphism, and hence so is the right hand vertical arrow. But this means precisely that we have (6), as desired. 


\subsection{Dual free products by the circle}

The following result, used above in the proof of Theorem 3.1, might be of some independent interest.

Theorem 3.4 Let $G$ be a compact connected Lie group and $T \leq G$ a maximal torus. We then have

$$
\mathbb{T} \hat{*} G=\langle\mathbb{T} \hat{*} T, G\rangle .
$$

Proof We have to argue that the Hopf algebra surjections

$$
\mathcal{O}(\mathbb{T}) * \mathcal{O}(G) \rightarrow \mathcal{O}(\mathbb{T}) * \mathcal{O}(T), \quad \mathcal{O}(\mathbb{T}) * \mathcal{O}(G) \rightarrow \mathcal{O}(G)
$$

do not factor through any proper Hopf quotient of $\mathcal{O}(\mathbb{T}) * \mathcal{O}(G)$. To that end, let

$$
\mathcal{O}(\mathbb{T}) \rightarrow \mathcal{O}(G) \rightarrow H
$$

be the smallest Hopf quotient factoring the two maps. Since all maximal tori of $G$ are mutually conjugate, we have factorizations

$$
\mathcal{O}(\mathbb{T}) * \mathcal{O}(G) \rightarrow H \rightarrow \mathcal{O}(\mathbb{T}) * \mathcal{O}\left(T_{i}\right)
$$

for every maximal torus $T_{i} \leq G$.

Consider a finite set of maximal tori $T_{i} \leq G, 1 \leq i \leq k$ such that the product

$$
T_{1} \times \cdots \times T_{k} \rightarrow G
$$

is onto. At the level of function algebras, this means that the iterated coproduct

$$
\Delta^{(k-1)}: \mathcal{O}(G) \rightarrow \mathcal{O}\left(T_{1}\right) \otimes \cdots \otimes \mathcal{O}\left(T_{k}\right)
$$

is an embedding.

The analogous iterated coproduct

$$
\Delta^{(k-1)}: \mathcal{O}(\mathbb{T}) * \mathcal{O}(G) \rightarrow\left(\mathcal{O}(\mathbb{T}) * \mathcal{O}\left(T_{1}\right)\right) \otimes \cdots \otimes\left(\mathcal{O}(\mathbb{T}) * \mathcal{O}\left(T_{k}\right)\right)
$$

lands inside the algebra $\mathcal{A}$ generated by the diagonal subalgebra

$$
\mathcal{O}(\mathbb{T}) \subset \mathcal{O}(\mathbb{T})^{\otimes k}
$$

and

$$
\mathcal{O}\left(T_{1}\right) \otimes \cdots \otimes \mathcal{O}\left(T_{k}\right)
$$

Now note that

$$
\mathcal{A} \cong \mathcal{O}(\mathbb{T}) *\left(\mathcal{O}\left(T_{1}\right) \otimes \cdots \otimes \mathcal{O}\left(T_{k}\right)\right)
$$

and hence (9) must be one-to-one because (8) is. Since on the other hand (9) factors through the quotient $\mathcal{O}(\mathbb{T}) * \mathcal{O}(G) \rightarrow H$, it follows, as desired, that this quotient is the identity. 


\section{References}

[1] Eiichi Abe. Hopf algebras, volume 74 of Cambridge Tracts in Mathematics. Cambridge University Press, Cambridge-New York, 1980. Translated from the Japanese by Hisae Kinoshita and Hiroko Tanaka.

[2] T. Banica. A note on the half-liberation operation. arXiv e-prints, March 2019.

[3] T. Banica. Compact quantum groups generated by their tori. arXiv e-prints, March 2019.

[4] T. Banica. Quantum groups under very strong axioms. arXiv e-prints, January 2019.

[5] Teodor Banica. Le groupe quantique compact libre U(n). Comm. Math. Phys., 190(1):143-172, 1997.

[6] Teodor Banica and Issan Patri. Maximal torus theory for compact quantum groups. Illinois J. Math., 61(1-2):151-170, 2017.

[7] A. Bhattacharya, M. Brannan, A. Chirvasitu, and S. Wang. Kirchberg factorization and residual finiteness for discrete quantum groups. ArXiv e-prints, December 2017.

[8] Angshuman Bhattacharya and Shuzhou Wang. Kirchberg's factorization property for discrete quantum groups. Bull. Lond. Math. Soc., 48(5):866-876, 2016.

[9] Michael Brannan, Benoît Collins, and Roland Vergnioux. The Connes embedding property for quantum group von Neumann algebras. Trans. Amer. Math. Soc., 369(6):3799-3819, 2017.

[10] Alexandru Chirvasitu. Residually finite quantum group algebras. J. Funct. Anal., 268(11):3508-3533, 2015.

[11] A. Connes. Classification of injective factors. Cases $I I_{1}, I I_{\infty}, I I I_{\lambda}, \lambda \neq 1$. Ann. of Math. (2), 104(1):73-115, 1976.

[12] Mathijs S. Dijkhuizen and Tom H. Koornwinder. CQG algebras: a direct algebraic approach to compact quantum groups. Lett. Math. Phys., 32(4):315-330, 1994.

[13] Pavel Etingof, Shlomo Gelaki, Dmitri Nikshych, and Victor Ostrik. Tensor categories, volume 205 of Mathematical Surveys and Monographs. American Mathematical Society, Providence, RI, 2015.

[14] Eberhard Kirchberg. Discrete groups with Kazhdan's property T and factorization property are residually finite. Math. Ann., 299(3):551-563, 1994.

[15] Susan Montgomery. Hopf algebras and their actions on rings, volume 82 of CBMS Regional Conference Series in Mathematics. Published for the Conference Board of the Mathematical Sciences, Washington, DC; by the American Mathematical Society, Providence, RI, 1993.

[16] David E. Radford. Hopf algebras, volume 49 of Series on Knots and Everything. World Scientific Publishing Co. Pte. Ltd., Hackensack, NJ, 2012.

[17] Moss E. Sweedler. Hopf algebras. Mathematics Lecture Note Series. W. A. Benjamin, Inc., New York, 1969. 
[18] Alfons Van Daele and Shuzhou Wang. Universal quantum groups. Internat. J. Math., 7(2):255263, 1996.

[19] Shuzhou Wang. Quantum symmetry groups of finite spaces. Comm. Math. Phys., 195(1):195211, 1998.

Department of Mathematics, University at Buffalo, Buffalo, Ny 14260-2900, USA

E-mail address: achirvas@buffalo.edu 